\title{
Advanced photoinjector experiment photogun commissioning results
}

F. Sannibale, * D. Filippetto, C. F. Papadopoulos, J. Staples, R. Wells, B. Bailey, K. Baptiste, J. Corlett, C. Cork, S. De Santis, S. Dimaggio, L. Doolittle, J. Doyle, J. Feng, D. Garcia Quintas, G. Huang, H. Huang, T. Kramasz, S. Kwiatkowski, R. Lellinger, V. Moroz, W. E. Norum, H. Padmore, C. Pappas, G. Portmann, T. Vecchione, M. Vinco, M. Zolotorev, and F. Zucca Lawrence Berkeley National Laboratory, 1 Cyclotron Road, Berkeley, California 94720, USA (Received 25 July 2012; published 3 October 2012)

\begin{abstract}
The Advanced Photoinjector Experiment (APEX) at the Lawrence Berkeley National Laboratory is dedicated to the development of a high-brightness high-repetition rate (MHz-class) electron injector for $\mathrm{x}$-ray free-electron laser (FEL) and other applications where high repetition rates and high brightness are simultaneously required. The injector is based on a new concept $\mathrm{rf}$ gun utilizing a normal-conducting (NC) cavity resonating in the VHF band at $186 \mathrm{MHz}$, and operating in continuous wave (cw) mode in conjunction with high quantum efficiency photocathodes capable of delivering the required charge at $\mathrm{MHz}$ repetition rates with available laser technology. The APEX activities are staged in three phases. In phase 0 , the $\mathrm{NC} \mathrm{cw}$ gun is built and tested to demonstrate the major milestones to validate the gun design and performance. Also, starting in phase 0 and continuing in phase I, different photocathodes are tested at the gun energy and at full repetition rate for validating candidate materials to operate in a high-repetition rate FEL. In phase II, a room-temperature pulsed linac is added for accelerating the beam at several tens of $\mathrm{MeV}$ to reduce space charge effects and allow the measurement of the brightness of the beam from the gun when integrated in an injector scheme. The installation of the phase 0 beam line and the commissioning of the VHF gun are completed, phase I components are under fabrication, and initial design and specification of components and layout for phase II are under way. This paper presents the phase 0 commissioning results with emphasis on the experimental milestones that have successfully demonstrated the APEX gun capability of operating at the required performance.
\end{abstract}

DOI: 10.1103/PhysRevSTAB.15.103501

PACS numbers: 29.25.Bx, 29.27.Bd, 29.27.Eg, 41.60.Cr

\section{INTRODUCTION}

The advent of free-electron laser (FEL) based x-ray light sources [1-4] represented a revolution in terms of capability and opportunities that such facilities offer to the user community. The respective $\sim 9$ and $\sim 3$ orders of magnitude increase in peak photon beam brightness and in time resolution with respect to ring based synchrotron radiation sources (so-called 3rd generation light sources) has opened the way to new classes of experiments not accessible before. Additionally, the recent demonstration of seeding schemes in existing FELs [4-6] shows the potential for great improvements in the control of the photon wavelength bandwidth, intensity, timing, and longitudinal coherence.

The next revolutionary leap forward will extend such performance from present repetition rates of order $100 \mathrm{~Hz}$ to $\mathrm{MHz}$ and beyond, allowing for experiments where large statistical samples are required, and dramatically decreasing the time required to perform experiments. A strong scientific case has been developed for such high repetition

\footnotetext{
*FSannibale@LBL.gov
}

Published by the American Physical Society under the terms of the Creative Commons Attribution 3.0 License. Further distribution of this work must maintain attribution to the author(s) and the published article's title, journal citation, and DOI. rate FELs [7], and the construction of several linac based $\mathrm{MHz}$ repetition rate facilities serving multiple independent FELs has been proposed [8-11].

In accelerator based light sources, the brightness of the photon beam depends directly on that of the electron beam. High beam brightness requires low emittances, and in 3 rd generation light sources, the minimum geometric emittance that the stored electron beam can achieve is determined (and limited) by equilibrium conditions that depend on the square of the beam energy and on the ring characteristics. In typical $\mathrm{x}$-ray FEL schemes, the electron beam passes through a linac only once and does not reach equilibrium, while the geometric emittance scales inversely with the beam energy. If the linac and transport lines are properly designed, their degradation of the emittance and hence of the brightness of the electron beam becomes minimal. Because of this, in an FEL the photon performance ultimately depends on the brightness of the electron gun, making the design and construction of highbrightness guns of fundamental importance.

The successful normal-conducting (NC) high-frequency ( $\gtrsim 1 \mathrm{GHz}$ ) high-brightness technology used in present low repetition rate x-ray FEL rf guns [12] cannot be scaled to repetition rates beyond $\sim 10 \mathrm{kHz}$ because the heat load due to Ohmic losses in the gun cavity becomes too large for being dissipated by the cooling system [13]. Many groups 
around the world are actively working on alternative electron gun schemes and technologies to achieve the required brightness at high repetition rates [14]. At the present time, despite some promising results [15], none has demonstrated the necessary set of requirements described in Ref. [16].

The Advanced Photoinjector Experiment (APEX) [17] at the Lawrence Berkeley National Laboratory is designed to fill that gap by developing a gun and an injector capable of the required performance. The gun, based on a novel concept $[18,19]$, has been fabricated and recently completed the first phase of its commissioning. All the performance milestones included in this part of the project were successfully achieved and this paper reports the results of the related tests.

\section{APEX, THE ADVANCED PHOTOINJECTOR EXPERIMENT}

APEX is an electron injector built around an rf photogun designed to operate electron beams with parameters in the range shown in Table I (even though operation of the gun outside the specified ranges is quite possible). The core of the gun is a NC copper rf cavity operating in continuous wave (cw) mode in the VHF band at $186 \mathrm{MHz}$ using reliable and mature mechanical and rf technologies. The frequency value is chosen to be close to either the 7th subharmonic of $1.3 \mathrm{GHz}$ or the 8th subharmonic of $1.5 \mathrm{GHz}$, making the gun operation compatible with both of the main superconducting electron linac technologies presently available [20,21].

Figure 1 shows a CAD cross section of the cavity with its main components, and Table II contains the VHF gun main design parameters selected to satisfy the requirements in Table I and in Ref. [16]. The resonant copper structure is surrounded by a stainless steel shell that ensures the necessary mechanical rigidity and the proper vacuum

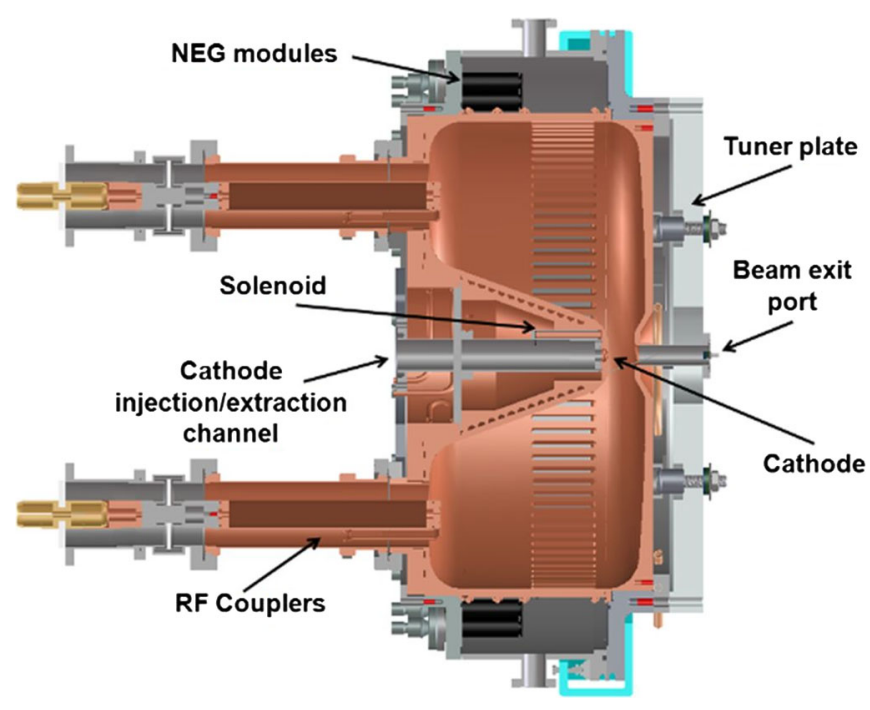

FIG. 1. APEX VHF gun cross section with main components.
TABLE I. Expected VHF gun beam parameter range.

\begin{tabular}{lc}
\hline \hline Parameter & Value \\
\hline Repetition rate & Up to few $\mathrm{MHz}$ \\
Nominal beam energy $(\mathrm{keV})$ & 750 \\
Charge per bunch $(\mathrm{pC})$ & $\sim 10-300$ \\
Normalized slice emittance $^{\mathrm{a}}(\mu \mathrm{m})$ & $\sim 0.2-0.6$ \\
Bunch length at the cathode $^{\mathrm{a}}$ (flattop, ps) & $\sim 5-50$ \\
\hline \hline
\end{tabular}

${ }^{\mathrm{a}}$ Larger values for larger charges/bunch.

envelope. No sliding tuner is present and the required frequency tuning is achieved by a mechanical system that slightly pushes or pulls the cavity wall at the beam exit port side. The rf power is supplied through two magnetic loop couplers diametrically opposed on the cathode back wall of the cavity. Figure 1 also shows the "bucking" solenoid (imbedded into the cavity nosecone) used to cancel any potential magnetic field at the cathode plane due to the fringe field of the first solenoid in the downstream beam line. A vacuum loadlock system, based on the Instituto Nazionale di Fisica Nucleare (INFN) design [22] (used at the FLASH and PITZ facilities in Germany) allows the replacement and/or the in situ conditioning of photocathodes without breaking the vacuum in the gun.

More details on the gun can be found elsewhere [17-19]; here we want just to remark that the two major goals targeted by the gun design are the cw operation, and the low-vacuum performance $\left(10^{-11}-10^{-9}\right.$ Torr) necessary to operate with acceptable lifetime high quantum efficiency (QE) semiconductor photocathodes sensitive to ion back bombardment and contamination. Such cathodes are required to generate the desired charge per bunch at high repetition rate with the power available by present laser technology.

The relatively low rf frequency choice for the VHF gun has addressed both of these needs. The larger resonating structure associated with the VHF frequency decreases the power density on the cavity walls to a level small enough to

TABLE II. VHF gun main design parameters.

\begin{tabular}{lc}
\hline \hline Parameter & Value \\
\hline Frequency $(\mathrm{MHz})$ & 186 \\
Operation mode & $\mathrm{cW}$ \\
Field @ cathode during emission $(\mathrm{MV} / \mathrm{m})$ & 19.47 \\
Ideal conductor quality factor, $Q_{0}$ & 30900 \\
Shunt impedance $(\mathrm{M} \Omega)$ & 6.5 \\
Nominal rf power for $Q_{0}(\mathrm{~kW})$ & 87.5 \\
Stored energy $(\mathrm{J})$ & 2.3 \\
Maximum surface field $(\mathrm{MV} / \mathrm{m})$ & 24.1 \\
Maximum wall power density $\left(\mathrm{W} / \mathrm{cm}^{2}\right)$ & 25.0 \\
Accelerating gap (cm) & 4 \\
Cavity inner diameter $(\mathrm{cm})$ & 69.4 \\
Cavity length $(\mathrm{cm})$ & 35.0 \\
Operating pressure $($ Torr $)$ & $\sim 10^{-11}-10^{-9}$ \\
\hline \hline
\end{tabular}




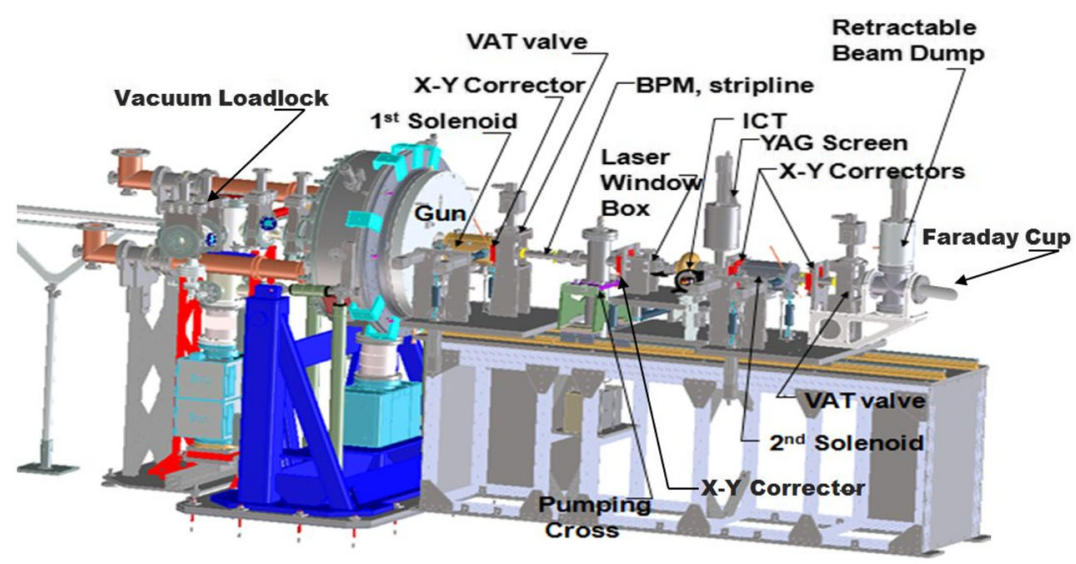

FIG. 2. APEX phase 0 layout. The about $2.5 \mathrm{~m}$ long beam line allows one to characterize and test cathodes at $\mathrm{MHz}$ repetition rates at the nominal gun energy of $750 \mathrm{keV}$.

permit $\mathrm{cw}$ operation with conventional cooling techniques while maintaining the high electric fields required for the high-brightness performance. Additionally, the long wavelength allows opening large slots visible on the cavity walls in Fig. 1 with negligible field distortion, thereby creating an extremely high vacuum conductance connecting to the pumping system located in the plenum around the cavity equator. Twenty $400 \mathrm{l} / \mathrm{s}$ commercial nonevaporable getter (NEG) pumps (SAES CapaciTorr®-D 400-2) ensures a formidable pumping of cathode contaminating molecules $\left(\mathrm{H}_{2} \mathrm{O}, \mathrm{O}_{2}, \ldots\right)$, while a $400 \mathrm{l} / \mathrm{s}$ ion pump connected to the bottom of the plenum removes noble gases and residual hydrocarbons.

The choice of the accelerating gradient for the VHF gun represents a tradeoff between contrasting requirements. From one side, beam dynamics considerations push towards higher gradients, while minimizing dark current (due to field emission) demands relatively low gradient. Mitigating dark current from the gun in a high repetition rate facility is of particular importance for avoiding undesired radiation losses along the main linac, and the risk of "quenching" downstream superconductive accelerating sections. Extensive beam dynamics simulations [23] showed that the gradient value in Table II for the VHF gun allows delivering the required brightness performance, and comparison with dark current vs gradient data for existing guns [24] jointly with initial dark current tracking studies for an FEL application [25] indicate also that the selected gradient should keep dark current at tolerable levels.

APEX is staged in three phases. Phase 0, illustrated in Fig. 2, consists of the VHF gun and a diagnostic beam line for cathode characterization. The primary scope of this phase is to commission the VHF gun and validate the technological choices used for it. A number of fundamental

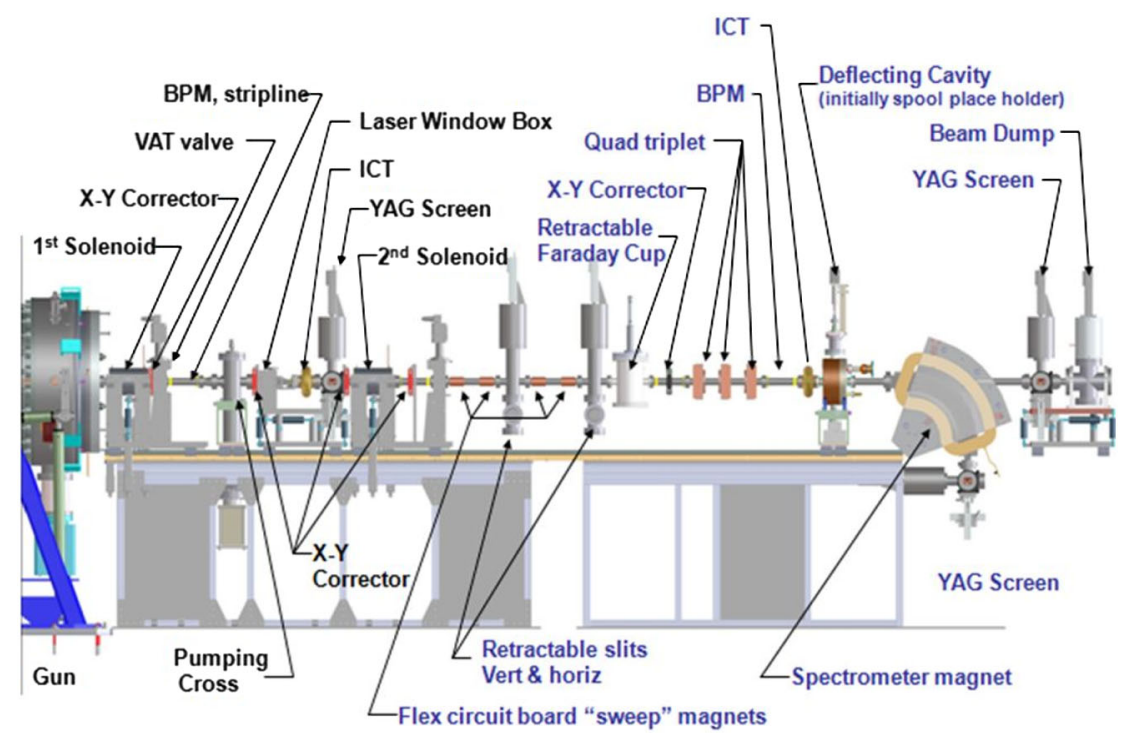

FIG. 3. APEX phase I layout. The addition of a diagnostic suite to the phase 0 beam line allows the 6D phase space characterization of the electron beam at $\mathrm{MHz}$ repetition rates at the nominal gun energy of $750 \mathrm{keV}$. 


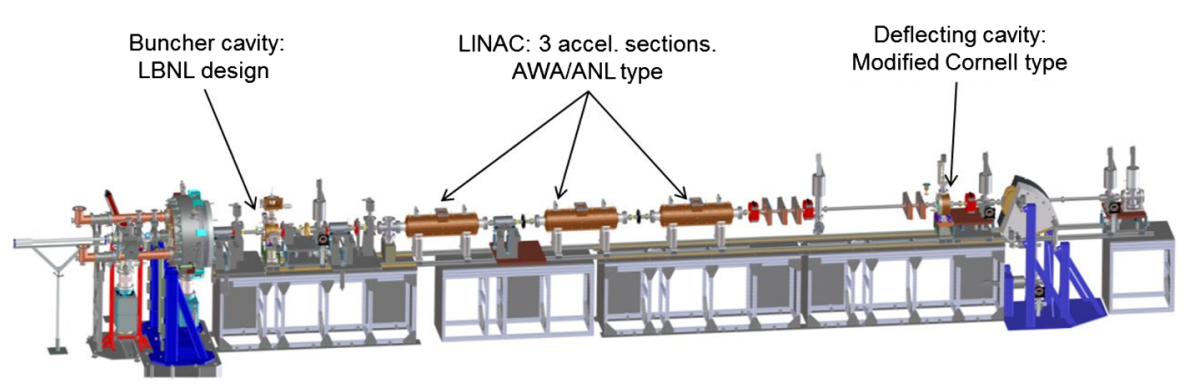

FIG. 4. APEX phase II layout. A pulsed room-temperature linac is added to accelerate the beam to the nominal energy of $30 \mathrm{MeV}$ to reduce space charge forces and perform beam brightness measurements. In this configuration, the total length of the accelerator is about $12 \mathrm{~m}$.

milestones need to be demonstrated in this critical phase, including full $\mathrm{cw}$ rf conditioning of the gun cavity, achievement of the design gradient at the cathode and associated beam energy at the gun exit, demonstration of the challenging vacuum performance, and characterization of dark current from the gun. Phase 0 also includes a photocathode $R \& D$ program in which different materials will be tested at $\mathrm{MHz}$ repetition rate at the gun energy to define the best choice for a high repetition rate $\mathrm{x}$-ray FEL.

In phase I, shown in Fig. 3, an electron beam diagnostic suite [26] is added to the phase 0 layout, to allow a full 6D characterization of the beam phase space at $\mathrm{MHz}$ repetition rate and at the nominal gun exit energy. The diagnostic suite includes among other components, a two-slit emittance measurement system (to measure the emittance in the space charge dominated regime), a transverse deflecting cavity (both based on a modified Cornell design [27,28]), and a spectrometer for slice emittance and energy spread measurements and full longitudinal phase space characterization. At the relatively low energy of the gun and for the typical charges per bunch used in FELs (hundreds of $\mathrm{pC})$, space charge forces are still dominant and the emittance compensation process is not completed yet, preventing the measurement of the final brightness performance. Nevertheless, the beam experiments during phase I will permit comparing measurements with simulation results verifying the intermediate injector performance at the gun energy, and the continuation of the photocathode studies.

In phase II, shown in Fig. 4, a $1.3 \mathrm{GHz}$ roomtemperature pulsed linac (using 3 ANL-AWA accelerating sections [29]) and a room-temperature $1.3 \mathrm{GHz}$ buncher cavity (a scaled version of the Advanced Light Source harmonic cavity [30]) are added to the phase I layout. The electron beam diagnostic suite of phase I (after some modification) is moved downstream of the linac. The new linac system will accelerate the beam up to $\sim 30 \mathrm{MeV}$ making space charge forces sufficiently small to perform reliable measurements of beam brightness while also compressing the bunch to the required length. A pulsed $L$-band linac system is chosen to allow realistic characterization of beam dynamics with components similar in rf fields and wakefields to superconducting cavities, while minimizing costs and remaining within the radiation safety envelope of the test area (the existing Beam Test Facility at the Advanced Light Source). This arrangement allows demonstration of the brightness performance of an injector based on the VHF gun, and brightness being a single bunch property, the limited repetition rate does not affect the scope of this phase.

Completion of phase II would present an opportunity for tests of accelerator components using the high-brightness beam and could include IR-FEL experiments using new scheme short period undulators [31] and/or electron diffraction imaging at high repetition rates.

Phase 0 beam line installation is completed, phase I components are under fabrication, and initial design and specification of components and layout for phase II are under way.

\section{APEX PHASE 0 COMMISSIONING RESULTS}

Currently, all phase 0 hardware (with the exception of the vacuum loadlock system and of the frequency tuning system actuators) has been installed. Figure 5 shows the beam line in the test area. The beam diagnostics included in the present layout allows for the measurement of beam

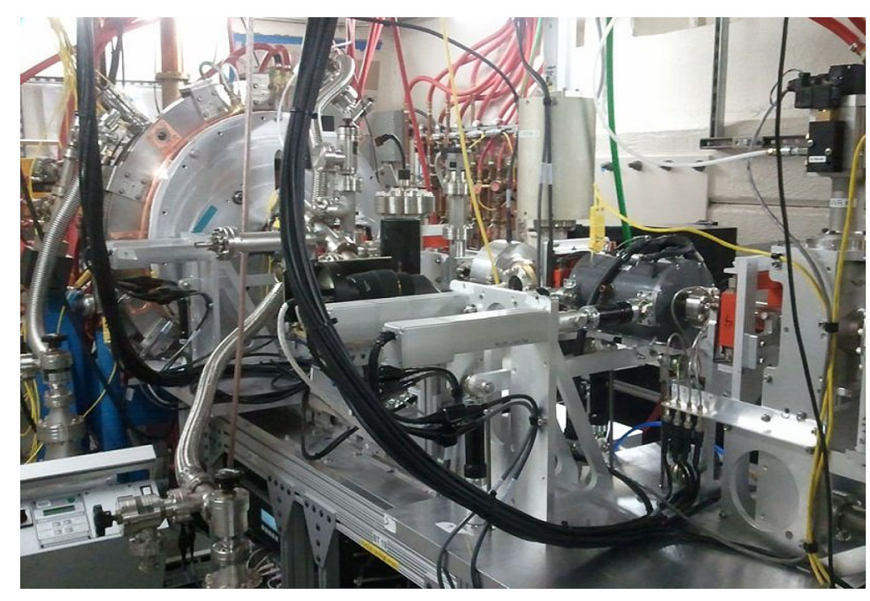

FIG. 5. APEX phase 0 installed in the Beam Test Facility at the Advanced Light Source. 
current, energy and transverse profile, and of cathode QE maps, intrinsic emittance, and lifetime [26].

The photocathode drive laser, developed in collaboration with Lawrence Livermore National Laboratory and UC Berkeley, has been installed and fully commissioned [32]. The IR pulses generated at $1064 \mathrm{~nm}$ by the $\mathrm{Yb}$-doped fiber laser are frequency converted to generate 532 and $266 \mathrm{~nm}$ in the 2nd and the 4th harmonic. This photon energy flexibility allows driving photoemission from various different cathode materials. The two main cathodes to be tested in the near future are $\mathrm{CsK}_{2} \mathrm{Sb}$ produced at Lawrence Berkeley National Laboratory (LBNL) [33,34], and $\mathrm{Cs}_{2} \mathrm{Te}$ developed in collaboration with INFN Milano LASA [22]. Both materials offer QEs $>1 \%$, with photoemission in the visible for the antimonide, and in the UV for the telluride. The available laser power at both harmonics is sufficient to drive photoemission in the cathodes at the required design charge ( $300 \mathrm{pC} /$ bunch) at $\mathrm{MHz}$ repetition rate. A copper photocathode in conjunction with a hydrogenated diamond amplifier will also be tested, in collaboration with Brookhaven National Laboratory [35].

The $120 \mathrm{~kW} 186 \mathrm{MHz} \mathrm{cw}$ rf source for the gun (fabricated by ETM Electromatic Inc.) is fully operational and reliably delivering the required power to the gun through two $60 \mathrm{~kW}$ tetrodes (Thales TH571B). The power level stability of the system is $\sim 10^{-3} \mathrm{rms}$ when controlled by a high level software feedback.

The EPICS based control system is in an advanced stage of implementation and permits the full control of the beam line components and acquisition of experimental data. High level macros developed in MatLab ${ }^{\circledR}$ on LINUX platforms allow for a flexible system operation and experiment control.

The FPGA-based low-level rf system (LLRF) is developed at LBNL and allows control of the rf system, the synchronization with the laser, and the tuning of the rf source frequency to follow the cavity frequency [36].

\section{A. VHF gun cavity low power rf tests}

In the summer of 2010, the first important experimental result for the project was achieved. The gun fabrication was completed and the low power rf tests were performed. The measured resonance frequency was in agreement with the expected value, and the measured cavity quality factor $Q$, see Fig. 6, was $\sim 86 \%$ of the ideal conductor case demonstrating the excellent quality of the cavity fabrication and the accuracy of the design model. With the measured $Q$ value, $\sim 100 \mathrm{~kW}$ of rf power are required to accelerate the beam to the nominal energy of $750 \mathrm{keV}$, well within the capability of the VHF rf source.

The frequency shift induced by pulling (or pushing) the cavity wall on the beam exit side, important for calibrating the action of the mechanical tuner, was also measured. Figure 7 shows the very first three runs of measurements. After some settling of the mechanical parts observable in

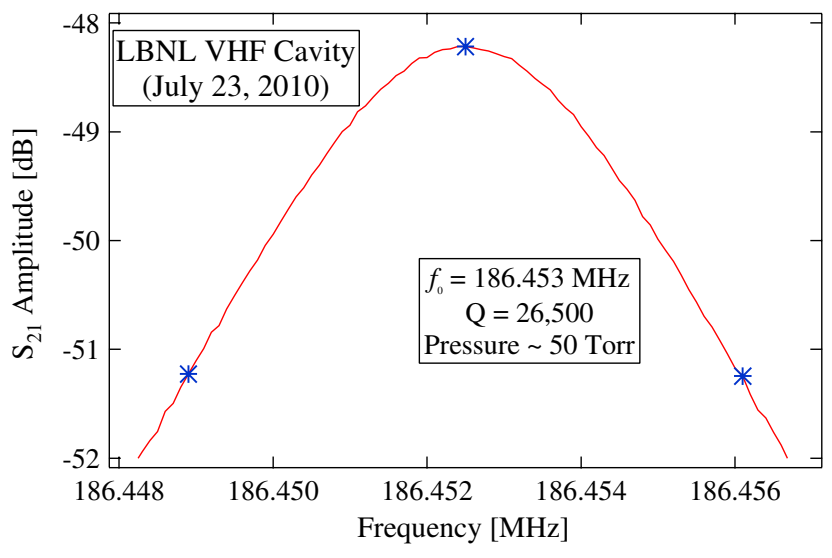

FIG. 6. Example of VHF gun quality factor measurement.

the first run, the second and third runs showed a linear and reproducible frequency dependence on the wall displacement. Linear fits of run 2 and run 3 points give a slope of $\sim 630 \mathrm{kHz} / \mathrm{mm}$, in good agreement with simulation predictions. The actual displacement (and hence the natural cavity frequency) will be remotely set by heavy duty piezomotors controlled by the LLRF system.

\section{B. Full power cw rf conditioning}

The second milestone for the project was achieved at the end of 2011 when the gun cavity was conditioned at the nominal power of $100 \mathrm{~kW}$ in $\mathrm{cw}$ mode after only $\sim 150$ integrated hours of conditioning. This important result confirmed the capability of the gun of operating at the required fields without rf breakdown. Continuous runs of many days with no faults showed a solid reliability of the rf system. As predicted by simulations, evidence of multipacting resonances in the cavity was detected at low power, and a wide multipacting-free region around the nominal operation point was confirmed. During the conditioning, the rf was first run in pulsed mode with $10 \%$ duty cycle. This approach allowed one to "jump" the low power

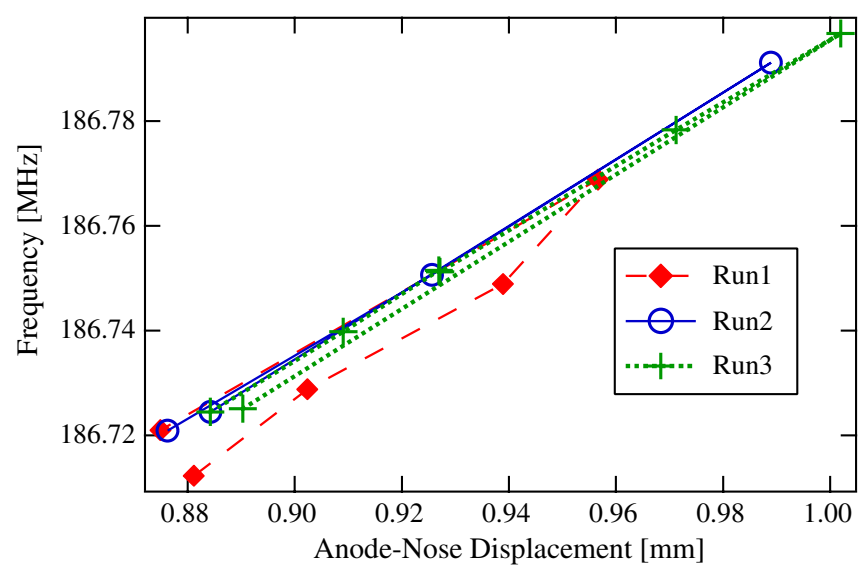

FIG. 7. Fundamental mode frequency shift vs "anode" wall cavity displacement induced by the tuner system. 
multipacting resonances by going directly to about half of the nominal power. From that point on, the peak power was first quickly increased up to the nominal value, and then the duty cycle was gradually increased up to $\mathrm{cw}$ operation.

Unexpected multipacting modes, in the $\sim 80 \mathrm{~cm}$ long (half an rf wavelength) rf feeder coaxial lines between the rf windows and the cavity couplers (indicated as "RF Couplers" in Fig. 1), were successfully cured by wrapping solenoids providing $\sim 50 \mathrm{G}$ field to this region of the coaxial lines.

\section{Dark current characterization}

Initial dark current characterization was performed in two phases. First, a coaxial Faraday cup was installed right at the gun beam exit pipe at $\sim 15 \mathrm{~cm}$ from the cathode. Figure 8 shows an example of a dark current measurement with the Faraday cup, and a Fowler-Nordheim fit of the data. The fitting function is averaged over the rf period to account for the time variable fields in the cavity [37]. With the gun running in $\mathrm{cw}$ at the nominal power, $\sim 8 \mu \mathrm{A}$ average current was measured. At a later time, the Faraday cup was moved to its present position at the end of the phase 0 beam line $\sim 2.5 \mathrm{~m}$ downstream of the cathode. In this new configuration, the dark current transported to the Faraday cup is partially collimated out by the vacuum chamber and the measured value drops down to less than a $\mu \mathrm{A}$ when the gun is operated in $\mathrm{cw}$ mode at the nominal power. Initial studies show that the measured values of dark current should be compatible with the operation of a high repetition rate $\mathrm{x}$-ray FEL [25].

By imaging the dark current on a yttrium-aluminumgarnet (YAG) screen using a solenoid in the transport line, it was observed that the field emission is mainly generated by few pointlike sources on the cathode plug area. In the present configuration, optimized for rf tests, a "dummy" molybdenum plug is installed instead of a real cathode. The better polishing level of a real cathode plug, and if required, a better cleaning technique to remove particulates

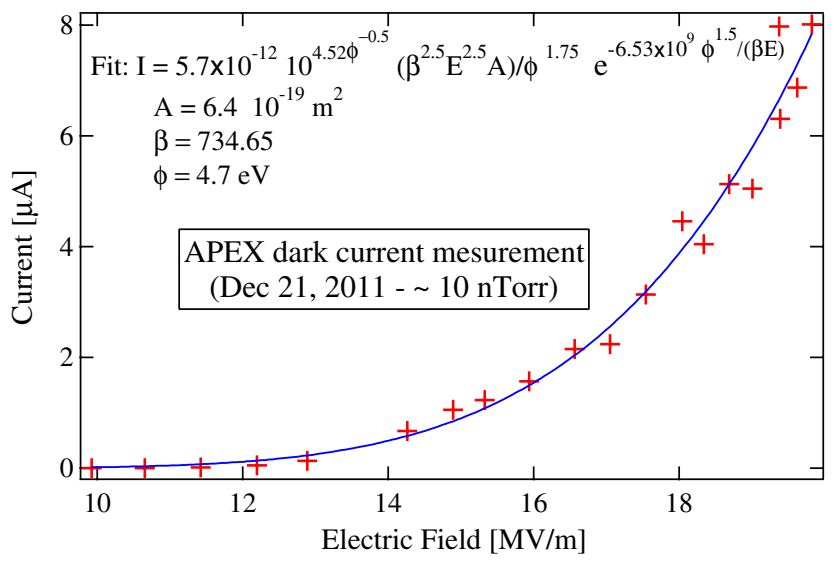

FIG. 8. Example of dark current measurement and FowlerNordheim fit.

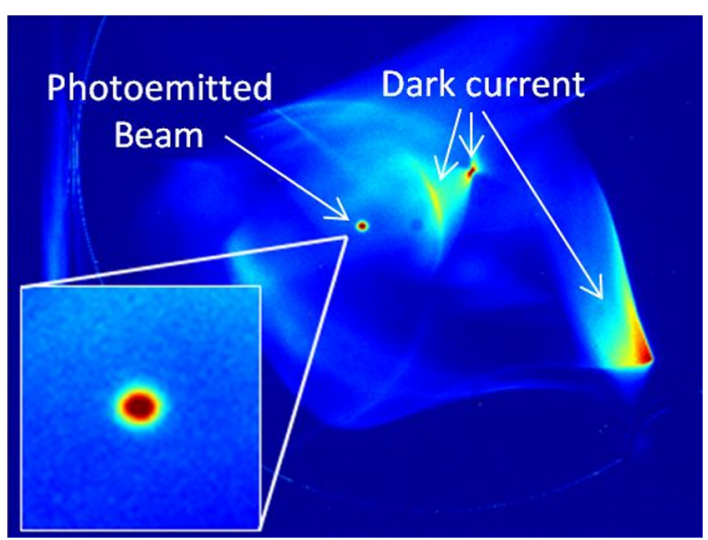

FIG. 9. Example of image of the photoemitted beam on the YAG screen in the phase 0 beam line.

[38], and/or a conditioning of the gun at higher rf fields [39] could potentially reduce the dark current intensity.

\section{First electron photoemitted beam at $1 \mathrm{MHz}$ repetition rate}

The dummy molybdenum cathode plug, used during this first commissioning, ensured the proper rf contact but presented a poor (estimated) QE of $\sim 10^{-6}$ at $266 \mathrm{~nm}$. With the available laser power and that QE, the expected photoemitted charge per bunch is $\sim 6 \mathrm{fC}$ that, with the $\mathrm{MHz}$ repetition rate, will generate an expected average current of $6 \mathrm{nA}$. Despite these challenging expectations, in March, 2012 the laser was synchronized with the gun rf by the LLRF system, and the photoemitted beam was visualized on the YAG screen of the phase 0 beam line.

Figure 9 shows an example of such a measurement.

In another measurement, by using a lock-in amplifier with the cathode drive laser ( $\mathrm{MHz}$ ) trigger as reference, an average current of $\sim 10 \mathrm{nA}$ was measured at the Faraday cup at the end of the beam line in fair agreement with the expected value.

\section{E. Demonstration of the electron beam design energy}

Another fundamental milestone for the project was achieved few days after the first photoemitted beam with the demonstration of the design electron beam energy. A schematic of the beam line components used during the measurement is shown in the bottom part of Fig. 10. The beam was focused on the beam line YAG screen by properly tuning the field in the first solenoid ("Sol.1" in the figure). A horizontal corrector $0.443 \mathrm{~m}$ upstream of the screen ("Hor. Corr. 2" in the figure) was subsequently energized at several different values and the beam centroid translation on the screen was recorded. An example of such a measurement is shown in the top part of Fig. 10. The slope of the fit is proportional to the particle momentum and the measured energy value was $745 \mathrm{keV}$ with a standard deviation of $41 \mathrm{keV}$, when the nominal rf power of 

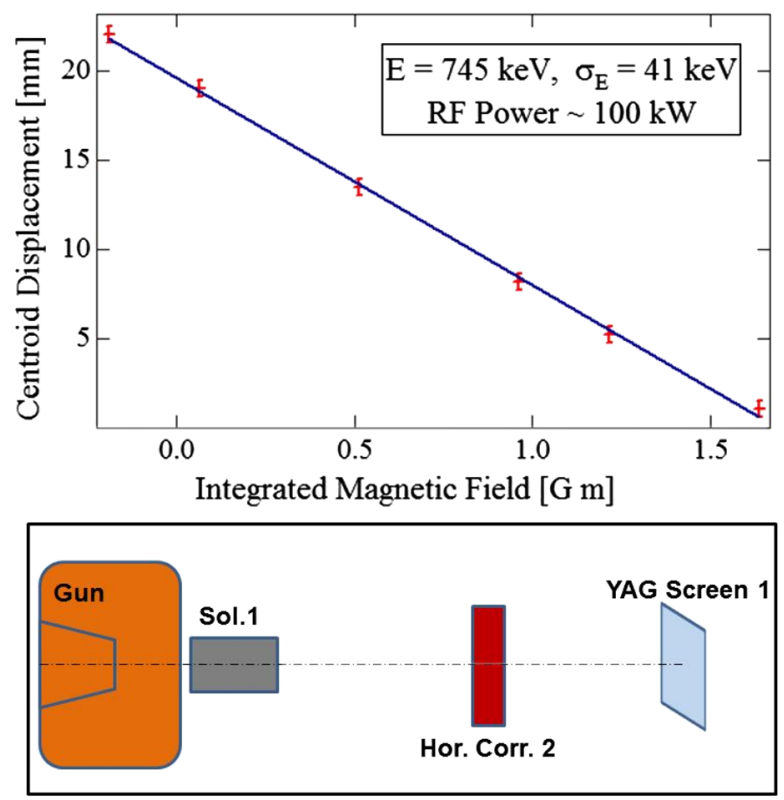

FIG. 10. Top: Example of photoemitted electron beam energy measurement. The design value for the nominal rf power of $100 \mathrm{~kW}$ is $750 \mathrm{keV}$ in excellent agreement with the measured value. Bottom: Schematics of the layout used for the measurement.

$100 \mathrm{~kW}$ was applied to the cavity. Such a value is in excellent agreement with the expected $750 \mathrm{keV}$, confirming again the capability of the gun to generate the required fields.

\section{F. Gun "rf baking" and vacuum performance}

The achievement of the required vacuum performance is of fundamental importance for the VHF gun to operate with semiconductor cathodes sensitive to contamination and ion back bombardment with acceptable lifetime.

The VHF gun has been designed to withstand high temperature vacuum baking $\left(\lessgtr 400^{\circ} \mathrm{C}\right)$. In the conventional scheme, the vacuum chamber to be baked is wrapped with heating tapes and maintained at temperatures higher than about $150^{\circ} \mathrm{C}$ for many hours to allow the desorption of gas molecules from the chamber walls. One limitation of such a scheme is that components included inside the external chamber under baking are heated only by conduction through their connection to the chamber itself. This situation can result in a difficulty in control of the actual temperature of these internal parts. In order to overcome this situation, we used for the VHF gun a technique that we named as "rf baking". In such a procedure, the water cooling of the gun is turned completely off and a few $\mathrm{kW}$ of average rf power are applied to the cavity. Without water, the temperature of the cavity walls rises and can be easily maintained at the desired level by adjusting the rf power level. In this way, the rf conducting surfaces that most need to be heated are brought to the desired temperature and a more effective vacuum baking is achieved.

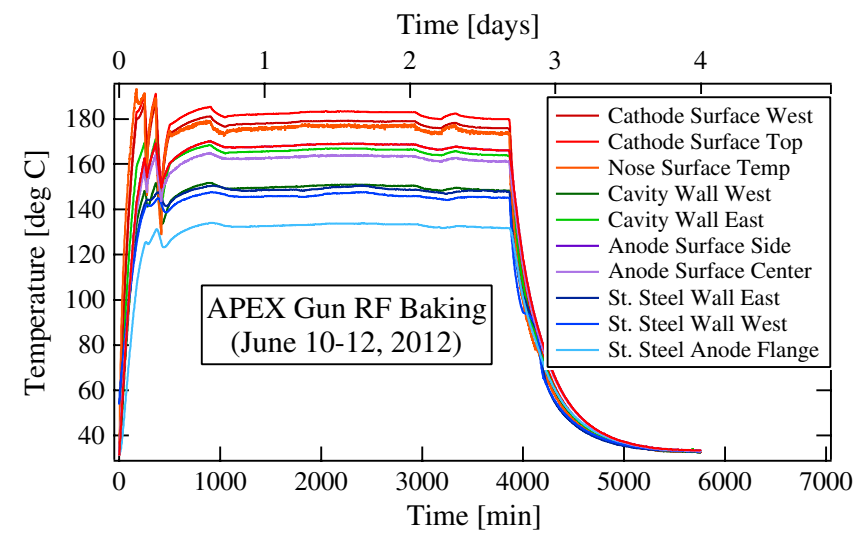

FIG. 11. Temperatures in different parts of the VHF gun during a $\sim 2.5$ day "rf baking" (see text for details).

Figure 11 shows the temperatures in different parts of the VHF gun during the "rf baking" demonstration experiment we performed. All the gun parts were stably maintained between 130 and $180^{\circ} \mathrm{C}$ for about 2.5 days with a turbomolecular pump on. At the end of the period, the rf was switched off, the cavity ion pump was switched on, and one out of the 20 NEG modules was activated.

Figure 12 shows a plot with the vacuum pressure inside the cavity (blue trace) and in proximity of the two rf windows in the couplers (red and green traces) with the rf power off and after cooling down from the baking shown in Fig. 11. The pressure value inside the cavity was below $5 \times 10^{-11}$ Torr $\left(\sim 6.7 \times 10^{-9} \mathrm{~Pa}\right)$ and a clear trend towards even lower pressures is clearly visible. The pressure inside the couplers, which were not significantly heated during the rf baking, was about 1 order of magnitude

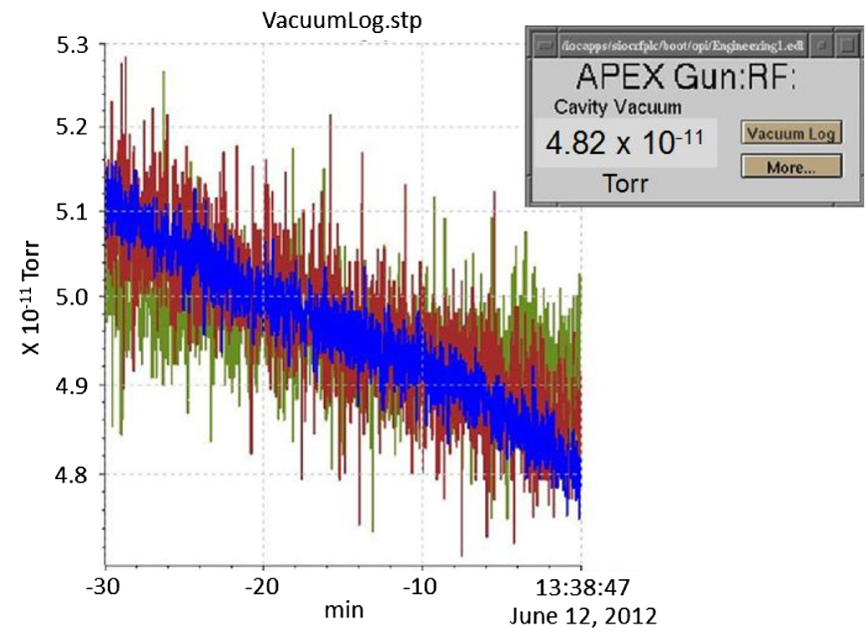

FIG. 12. Plot of the vacuum pressure inside the gun cavity after the rf baking shown in Fig. 11. The vertical scale is associated with the blue trace (pressure inside the cavity). The red and green traces (pressures inside the rf feeders) have different scales, and their values were about 1 order of magnitude higher than in the cavity. 
higher than in the cavity (the traces in Fig. 12 have different scales). Preliminary data show a typical pressure increase by a factor 3 to 4 when the nominal rf power is applied to the cavity.

The described rf baking procedure will be repeated after the installation of the vacuum loadlock and a larger number of NEG pumps will be activated to achieve the ultimate vacuum performance of the gun.

\section{CONCLUSIONS AND FUTURE PLANS}

The commissioning of the APEX photogun has been completed. The fundamental milestones for this phase of the APEX project have been successfully demonstrated showing the capability of the gun to generate $\mathrm{MHz}$ repetition rate electron beams at the design energy. An excellent base vacuum pressure in the gun has been also achieved paving the way for the tests of semiconductor photocathodes as scheduled in the next phases of the project.

Additional future activities include the addition of a beam diagnostic suite to perform a $6 \mathrm{D}$ phase space characterization of the electron beam first at the gun energy, and subsequently at several tens of $\mathrm{MeV}$ for the final brightness performance demonstration of the gun when integrated in a full injector scheme.

It is worth remarking that, in principle, repetition rates as high as the rf frequency can be achieved by the VHF gun, and that the actual repetition rate is limited by the available laser power used to drive the photocathode. Modified versions of the gun resonating at higher frequencies ( $\lesssim 700 \mathrm{MHz}$ ) may be used for energy recovery linac applications.

\section{ACKNOWLEDGMENTS}

The authors want to thank John Byrd, Peter Denes, Paul Emma, David Robin, and the AFRD, ALS, and Engineering Divisions for the continuous support during the various phases of the project, and Marco Venturini, Ji Qiang, and Weishi Wan for useful discussions. The authors want also to express their appreciation to Ali Nassiri and Conor Pogue for their helpful participation in some of the commissioning shifts. This work was supported by the Director of the Office of Science of the U.S. Department of Energy under Contract No. DE-AC02-05CH11231.

[1] P. Emma et al., Nature Photon. 4, 641 (2010).

[2] W. Ackermann et al., Nature Photon. 1, 336 (2007).

[3] H. Tanaka, in Proceedings of IPAC11, San Sebastian, Spain (EPS-AG, Spain, 2011), p. 21.

[4] S. Di Mitri et al., in Proceedings of IPAC11, San Sebastian, Spain (Ref. [3]), p. 918.

[5] P. J. Emma et al., in Proceedings of IPAC12, New Orleans, LA, 2012.

[6] S. Ackermann et al., in Proceedings of IPAC11, San Sebastian, Spain (Ref. [3]), p. 923.
[7] See, for example, E. Arenholz et al., in Proceedings of the Science for a New Class of Soft X-Ray Light Sources Workshop, Berkeley, CA, 2007 (LBNL Report No. LBNL-1034E, 2008).

[8] J. Corlett et al., Synchrotron Radiat. News 22, 25 (2009).

[9] J. Bisognano et al., in Proceedings of the 23rd Particle Accelerator Conference, Vancouver, Canada, 2009 (IEEE, Piscataway, NJ, 2009), p. 109.

[10] R. Bartolini et al., in Proceedings of the 31st International Free Electron Laser Conference (FEL 09), Liverpool, UK (STFC Daresbury Laboratory, Warrington, 2009), p. 480.

[11] K.-J. Kim, S. Reiche, and Y. Shvydko, Phys. Rev. Lett. 100, 244802 (2008).

[12] See, for example, C. Limborg-Deprey, D. Dowell, J. Schmerge, Z. Li, and L. Xiao, Report No. LCLS TN-05-3, 2005; S. Rimjaem et al., in Proceedings of the 31st International Free Electron Laser Conference (FEL 09), Liverpool, UK (Ref. [9]), p. 251.

[13] J.W. Staples, S.P. Virostek, and S.M. Lidia, in Proceedings of the 2004 European Particle Accelerator Conference (EPS-AG, Lucerne, Switzerland, 2004, p. 473.

[14] See, for example, F. Sannibale, in Proceedings of IPAC12, New Orleans, LA, 2012Proceedings of IPAC12, New Orleans, LA, 2012, p. 4160, and references therein.

[15] B. M. Dunham et al., in Proceedings of IPAC12, New Orleans, LA, 2012, p. 20.

[16] F. Sannibale, D. Filippetto, and C.F. Papadopoulos, J. Mod. Opt. 58, 1419 (2011).

[17] F. Sannibale et al., in Proceedings of the 2010 FEL Conference (Malmö, Sweden, 2010), p. 475.

[18] J. W. Staples, F. Sannibale, and S. Virostek, CBP Tech Note No. 366, 2006.

[19] K. Baptiste, J. Corlett, S. Kwiatkowski, S. Lidia, J. Qiang, F. Sannibale, K. Sonnad, J. Staples, S. Virostek, and R. Wells, Nucl. Instrum. Methods Phys. Res., Sect. A 599, 9 (2009).

[20] C. Reece et al., in Proceedings of the 1995 Particle Accelerator Conference and International Conference on High Energy Accelerators (IEEE, Dallas, TX, 1995), p. 1512 .

[21] B. Aune et al., Phys. Rev. ST Accel. Beams 3, 092001 (2000).

[22] P. Michelato, C. Pagani, and D. Sertore (private communication).

[23] C. F. Papadopoulos et al., in Proceedings of the 2010 FEL Conference (Malmö, Sweden, 2010), p. 479.

[24] See for example: L. Monaco et al., in Proceedings of the 10th European Particle Accelerator Conference, Edinburgh, Scotland, 2006 (EPS-AG, Edinburgh, Scotland, 2006), p. 2493; D. H. Dowell et al., in Proceedings of the 2007 Particle Accelerator Conference, Albuquerque, New Mexico (IEEE, New York, 2007), p. 1299.

[25] C. A. Steier et al., in Proceedings of IPAC12, New Orleans, LA, 2012, p. 1695.

[26] D. Filippetto et al., in Proceedings of the 2011 Particle Accelerator Conference, NY, USA (IEEE, New York, 2011), p. 1903.

[27] I. V. Bazarov, B. M. Dunham, Y. Li, X. Liu, D. G. Ouzounov, C. K. Sinclair, F. Hannon, and T. Miyajima, J. Appl. Phys. 103, 054901 (2008). 
[28] S. Belomestnykh, I. Bazarov, V. Shemelin, J. Sikora, K. Smolenski, and V. Veshcherevich, Nucl. Instrum. Methods Phys. Res., Sect. A 614, 179 (2010).

[29] J. Power et al., in Proceeding of IPAC10 (ICR, Kyoto, Japan, 2010), p. 4310.

[30] R. A. Rimmer et al., in Proceeding of EPAC98, Stockholm, Sweden (IOP, London, 1998), p. 1808.

[31] M. Yoon, D. Filippetto, C. F. Papadopoulos, C. Pellegrini, G. Penn, S. Prestemon, and F. Sannibale, Nucl. Instrum. Methods Phys. Res., Sect. A 660, 138 (2011).

[32] J. Feng et al., in Proceedings of the 2011 Particle Accelerator Conference, NY, USA (Ref. [26]), p. 2537.

[33] T. Vecchione, I. Ben-Zvi, D. H. Dowell, J. Feng, T. Rao, J. Smedley, W. Wan, and H. A. Padmore, Appl. Phys. Lett. 99, 034103 (2011).
[34] T. Vecchione et al., in Proceedings of IPAC12, New Orleans, LA, 2012, p. 655.

[35] I. Benz-vi, T. Rao, J. Smedley, and T. Xin (private communication).

[36] G. Huang et al., in Proceedings of IPAC12, New Orleans, LA, 2012, p. 3488.

[37] See, for example, J.W. Wang, Ph.D. thesis [Report No. SLAC-339 UC-28 (A), 1989].

[38] See, for example, D. Reschke et al., in Proceedings of the 2007 SRF Conference (Beijing, China, 2007), p. 239.

[39] See, for example, J. Norem, V. Wu, A. Moretti, M. Popovic, Z. Qian, L. Ducas, Y. Torun, and N. Solomey, Phys. Rev. ST Accel. Beams 6, 072001 (2003). 